\title{
CONSIDERACIONES TEORICAS SOBRE LAS GENERACIONES POLÍTICAS
}

\author{
Maria Fatima Pinho de Oliveira ${ }^{1}$
}

\section{Resumen}

El presente estudio se basa desde una perspectiva sociológica y politológica en describir teóricamente diversas acepciones sobre la generación política. En los partidos políticos de diferentes países, actúa el binomio: democracia representativa y participación social, demostrando una débil capacidad de conducción de la ciudadanía organizada, que ha traído como consecuencia que las instituciones públicas y los partidos políticos pierden legitimidad y confianza en los ciudadanos, generando tensiones internas entre jóvenes militantes líderes de movimientos estudiantiles y partidos políticos; estos movimientos conducen a una generación que busca la institucionalización del poder, que significa, una oportunidad de renovación de la política. Se utiliza el método hipotético-deductivo con la respectiva revisión bibliográfica para el desarrollo de la investigación. Varios autores refieren el concepto de generación desde el siglo XIX: Comte y Dilthey, Mannheim, y Ortega, Gasset y Gramsci. Se concluye que la generación política se caracteriza por un cambio drástico en el entorno de las relaciones de poder, que afecta o marca distintivamente a un grupo con respecto a sus predecesores, es decir, son contemporáneos.

Palabras Clave: Generaciones Políticas ; Cohortes; Grupos Etarios; Participación Social.

\section{INTRODUCCIÓN}

La modernización del estado y la legitimidad de la gobernabilidad democrática dependen de la capacidad del sistema político de gobierno de conjugar de la representación de los partidos políticos y los intereses ciudadanos en los diferentes territorios.

En los diferentes Estados y sus partidos políticos, pese a las altas expectativas del binomio democracia representativa y participación social han demostrado durante la última década una débil capacidad de conducción de la ciudadanía organizada. Contrariamente, la fórmula no da los resultados esperados, la institucionalidad público estatal y los partidos políticos carecen de la legitimidad y confianza de sus ciudadano.

Las tensiones internas entre los jóvenes militantes líderes de movimientos estudiantiles y sus bases sociales dan cuenta de la importante resistencia que se está generando hacia la conducción de los partidos políticos.

\footnotetext{
${ }^{1}$ Especialista y MSc. en Derecho Penal, y Doctoranda en Derecho Constitucional. Profesora Agregada de la Universidad Simón Bolívar del area de Comercio Exterior y Administración Aduanera. Coordinadora de Ciencias Sociales y Administrativas del Decanato de Investigación y Desarrollo de la USB. Docente investigadora y Arbitro de revistas internacionales en temas de Derecho y Politica. E-mail: mpinho@usb.ve
} 
Estos movimientos conducen a una generación que busca referentes fuera de toda forma de institucionalización del poder lo que si bien significa, una oportunidad de renovación de la política acarrea también el riesgo de polarización ideológica, caudillismo e ingobernabilidad civil.

Para las corrientes sociológicas de tipo estructural, como el marxismo o el funcionalismo, la evolución histórica de las generaciones no dependía de un acontecimiento determinante, sino de complejos procesos económicos, sociales o culturales que daban sentido a la identidad compartida por un conjunto más o menos extenso de individuos que resultaban contemporáneos y coetáneos. En esta perspectiva, las fronteras generacionales no responderían de forma estricta a factores biológicos, sino a circunstancias socio históricas de carácter estructural.

En el período de entreguerras, pioneros de la «teoría generacional» como

François Mentré, José Ortega y Gasset o Karl Mannheim pusieron énfasis respectivamente en los estados mentales (sentimientos y creencias) compartidos, en una común adscripción intelectual o en procesos de construcción de una identidad por sensibilidades, afinidades, proyectos e intereses colectivos, fruto de una experiencia vital coincidente durante los años de formación. (Mentré, 1920; Ortega y Gasset, 1923 y 1933 y Mannheim, 1952).

En un trabajo pionero que aún hoy resulta imprescindible para abordar el estudio del problema de la caracterización socio histórica del hecho juvenil, Mannheim destacaba que la dinámica generacional dependía del contexto social específico, y no al revés. De forma que la contemporaneidad no era un mero asunto de coincidencia cronológica (coetaneidad), sino un sentimiento de pertenencia basado en experiencias históricas compartidas, lo que implicaba similares percepciones de la realidad y determinados tipos de actuación considerados históricamente relevantes.

De acuerdo con las ideas de Ortega y Mannheim, una generación sería «un grupo de personas que, siendo contemporáneas y coetáneas, presentan cierta relación de coexistencia, es decir, que tienen intereses comunes, inquietudes analógicas o circunstancias parecidas»: tener la misma edad, estar situado en el mismo escenario temporal e histórico y mostrar perspectivas vitales semejantes (Zarco y Orueta, 1998, p. 109).

Una interpretación pionera del problema de la inserción social de la juventud fue la implícita en el análisis freudiano de la formación de la identidad en la adolescencia, momento en el que tiene lugar la conciliación o el conflicto entre los roles asignados a la juventud y otros nuevos que surgen al acercarse a la edad adulta.

Los jóvenes necesitados de autoafirmación a través del reconocimiento consciente o inconsciente de una autoridad son más proclives a integrarse en grupos cerrados, cuya organización formal les proporciona fortaleza y ánimo

Un segundo tipo de aproximación social al problema es la que pone el énfasis en los cambios sociales no rutinarios y en los conflictos en el sistema sociopolítico como fruto de la diferenciación estructural y la vol.10, nº.01, Rio de Janeiro, 2017.pp. 82-94 
modernización; las inconsistencias sociales y la discontinuidad de valores surgidas en una sociedad sometida a un proceso de cambio traumático influyen en la conciencia de la juventud, que se muestra más receptiva a las posibilidades y a las oportunidades de protestar contra las condiciones sociales creadas por sus mayores.

Algunos autores rechazan por excesivamente simplificadora la idea de la

existencia de una generación «joven»y otra «vieja» como contendientes exclusivos y excluyentes en el proceso político, de la misma forma en que Marx

esquematizó la lucha de clases en la moderna sociedad industrial como un enfrentamiento bipolar entre clases poseedoras y clases desposeídas (Zarco y Orueta, 1998, p.110).

Por lo interesante del tema, el presente estudio enfoca unas acepciones históricas sobre el concepto de generación y los diferentes postulados sostenidos por autores reconocidos.

\section{BREVES ACEPCIONES HISTÓRICAS}

Se puede decir que el concepto de generación, desde el punto de vista sociológico, data del siglo XIX, referenciados por Comte y Dilthey, pero desde el punto de vista moderno se hace referencia a Mannheim, considerado el fundador del enfoque moderno de las generaciones, así como las ideas de Ortega, Gasset y Gramsci, llegando a los años 90 por las teorías de Abrams. La teoría positivista de Comte y la histórica - romántica de Dilthey fueron tomadas por Mannheim en sus reflexiones sobre las generaciones, los otros autores incluyendo este último son referencias obligadas para un análisis social e histórico. (LECCARDIY FEIXA, 2011).

El postulado de Comte (1830-1857) estaba basado en el progreso y la sucesión de las generaciones, medidas por el tiempo medio necesario para la sustitución de una generación por otra (con un aproximado de 30 años). La palabra clave de la objetividad histórica es la continuidad. Para Auguste Comte, el progreso es el resultado del equilibrio entre los cambios producidos por las nuevas generaciones y la estabilidad mantenida por las generaciones anteriores; propuso una ley general sobre el ritmo de la historia. Las leyes biológicas, relacionadas con la duración media de vida y la sucesión de las generaciones marcan la "objetividad" de este ritmo. (LECCARDIY FEIXA, 2011).

Para Dilthey lo que importa es la calidad de los vínculos que unen a los componentes de una generación. Dilthey (1883-1989) argumentó que la cuestión de las generaciones requería del análisis de un tiempo de experiencia mensurable solamente en términos cualitativos. Por lo cual la generación consistía en personas que compartín el mismo conjunto de experiencias, la misma "calidad de tiempo"; dichas experiencias situadas históricamente determinan la pertenencia a una generación ya que constituyen la existencia humana; difería de Comte ya que sostenía que el tiempo de la naturaleza, a diferencia del tiempo humano, está constituido por una serie de momentos discontinuos de igual valor sin una estructura que consiste en pasado, presente y futuro. La 
naturaleza no tiene historia, y por tanto, no tiene sentido desde un punto de vista teórico. (LECCARDI Y FEIXA, 2011).

Para Mannheim (1928-1952), en el desarrollo de su teoría de las generaciones, las consideraba como dimensiones analíticas útiles para el estudio, tanto de las dinámicas del cambio social, como para los estilos de pensamiento y la actitud de la época. Se basaba en la colisión entre el tiempo biográfico y el tiempo histórico. Las generaciones podían ser consideradas como el resultado de las discontinuidades históricas, es decir, del cambio. Para Mannheim, la generación no es compartir la fecha de nacimiento, sino esa parte del proceso histórico que los jóvenes de igual edad-clase comparten, lo que el autor denomina la generación en sí; donde los dos componentes fundamentales son el vínculo generacional (acontecimientos que marcan un antes y un después) y las unidades generacionales (quienes elaboran vínculos diferentes de conformidad a los grupos a que pertenecen). (LECCARDI Y FEIXA, 2011).

El sociólogo inglés Philip Abrams (1982) cincuenta años después de la teoría original de Mannheim profundizó y expandió la noción histórico-social de la generación, relacionándola con la noción de identidad. Su intención era dilucidar la estrecha relación entre el tiempo individualy el tiempo social, enfatizando su afiliación conjunta al registro de la historia. Su punto de partida era su convicción de que la individualidad y la sociedad se construyen socialmente. Por lo tanto, es necesario analizar sus interconexiones y, simultáneamente, sus intercambios a lo largo del tiempo. La identidad — considerada como el vínculo entre las dos dimensiones del individuo y la sociedad - debe estudiarse dentro de un marco de referencia histórico-social. Para Abrams, una generación en el sentido sociológico es el período de tiempo durante el cual una identidad se construye sobre la base de los recursos y significados que socialmente e históricamente se encuentran disponibles. Por lo tanto no existe un tiempo normalizado con el cual medir o predecir su ritmo. Desde un punto de vista sociológico, una generación puede durar diez años, o puede durar varios siglos tal como sucedió en las sociedades premodernas. Indicó que cuando grandes acontecimientos históricos —o más frecuentemente, procesos lentos, no catastróficos de naturaleza económica, política y cultural — vacían de sentido el sistema previo y las experiencias sociales que se le asocian. (LECCARDI Y FEIXA, 2011).

\section{COHORTES O GRUPOS ETARIOS}

La edad es una característica individual, fundamental para el estudio de las actitudes políticas en la medida en que, entre otras cosas, asigna un rol y un estatus en el sistema social (JUSTEL, 1992). Donde algunos grupos de edad manifestarían ciertas tendencias políticas con mayor propensión que otros. Pero basados en esta variable de la edad se pueden encontrar dos defectos: el efecto del ciclo vital y el efecto de las cohortes.

El ciclo vital está ligado a los cambios económicos y sociales esperables en todos los individuos a medida 
que estos cumplan años. Concretamente, según los resultados reflejados en la literatura especializada, edad e interés mantendría una relación de tipo cuadrático por la que tanto los más jóvenes como los más mayores estarían menos interesados (en la política) que las personas de mediana edad. (Verba y Nie, 1972; Milbrath y Goel, 1977; Marsh et al, 2007, citado por GALAIS, 2012:87).

Los jóvenes son dependientes de sus padres, por lo que no tienen incentivo para informarse sobre las políticas públicas que los afectan como responsables de un hogar e hijos. (SAPIRO, 1994; STOKER y JENNINGS, 1995). No han experimentado estímulos políticos ni han entrado a formar parte de sus redes sociales (NIE et al.1996), lo que les haría sentirse más ajenos a los procesos políticos que los individuos adultos. En definitiva, los jóvenes presentarían una escasa propensión a interesarse por la política en comparación a los adultos, cosa que solo mejoraría al madurar.

Por otra parte también los más mayores presentarían bajos niveles de interés por la política. En un principio las teorías sobre la desvinculación política de los ancianos sugerirían que estos, independientemente de sus recursos materiales o de su salud, se desconecten de sus aficiones y militancias como una manera de prepararse para la desconexión de otras redes sociales y familiares y de minimizar el impacto negativo que tendrá su desaparición sobre la sociedad. (Cummings y Henry, 1961; citado por GALAIS, 2012:88).

El segundo efecto que operaria tras la variable edad estaría ligado a la socialización política y se conoce como "efecto cohorte". La teoría de la socialización política sugiere que los individuos interiorizan normas, valores y actitudes principalmente antes de su vida adulta (Markus, 1986; Deli Carpini, 1989; Stoker y Jennings, 2008); están bajo la influencia de los agentes clásicos de la socialización (familia, escuela), pero también de los discursos políticos y temas que dominan la agenda política (BEAN, 2005).

Como consecuencia, observaríamos patrones de similitud en el interés por la política de los individuos que nacieron en el mismo periodo y crecieron bajo las mismas circunstancias políticas, económicas y sociales y de diferencia entre individuos nacidos en distintas épocas (GLENN, 2005).

En este sentido se le denomina cohorte a cada uno de estos grupos de población nacidos en un determinado periodo y, por tanto, influenciados en sus valores, actitudes políticas por un mismo conjunto de factores sociales, históricos o políticos (GALAIS, 2012: 88).

A diferencia de la edad, las cohortes son un factor de cambio cultural; puesto que si el contexto en que crecieron los ciudadanos de distintas épocas es diferente, sus creencias y orientaciones políticas también lo serán y se producirá un efecto de sustitución o renovación cultural a medida que los ancianos mueran dejando paso a las nuevas generaciones (Campbell et al., 1960; Butler y Stokes, 1974; Inglehart, 1990). Por esto, los análisis de las cohortes permiten aproximarse a la continuidad y al cambio cultural (MASON Y WOLFINGER, 2001).

Si se interpreta el interés por la política como una manifestación de implicación política y predisposición a la participación, se podría considerar que aquellas cohortes crecidas en épocas de abundancia serán más vol.10, nº. 01, Rio de Janeiro, 2017.pp. 82-94 
propensas a interesarse que el resto. (GALAIS, 2012: 89).

Otra manifestación del grado de modernización y desarrollo social es el acceso a la educación, un factor que puede considerarse un indicador del lugar del individuo en la distribución del logro educativo, y por tanto, de su estatus (NIE et al., 1996). En cualquiera de los supuestos, la educación se relaciona positivamente con el interés por la política en el plano individual (VAN DETH, 2000).

Finalmente, es igualmente importante tener en cuenta los procesos políticos que tuvieron lugar cuando los individuos estaban desarrollando sus orientaciones políticas. Estos sucesos pueden ser eventos especialmente remarcables, como escándalos, guerras o cambios institucionales profundos (Delli Carpini, 1989; Bartels, 2001), pero también elecciones (Freie, 1997; Blais y Rubenson, 2012; Van der Eijk y Franklin, 2009). Algunas de sus características y el hecho mismo de su celebración podrían fomentar la implicación política de los ciudadanos que las experimentaron durante el resto de su vida.

\section{GENERACIONES POLITICAS}

Una generación política, desde una perspectiva sociológica y politológica, es una cohorte o grupo etario cuyos integrantes conviven durante una época determinada (la adolescencia o adultez temprana) en un cambio drástico en el entorno de las relaciones de poder, que los afecta o marca distintivamente con respecto a sus predecesores, es decir, son contemporáneos. (KOENEKE, 2007a).

En otras palabras, la generación política es producto de un cambio drástico en el entorno de las relaciones de poder, que afecta o marca distintivamente a individuos jóvenes; donde la familia y la escuela juegan un papel fundamental como agentes de educación y formación. Es por ello que la "generación política" queda restringido al surgimiento de grupos etarios con inclinaciones marcadamente diferentes a las de sus predecesores. (KOENEKE, 2007a).

Las generaciones políticas, surgen como resultado de eventos críticos que tienen un marcado impacto sobre las orientaciones y la visión del mundo de adolescentes y adultos jóvenes, con lo que se debilita o fractura el proceso gradual de socialización o aculturación. (KOENEKE, 2007a).

Se puede afirmar que una generación política existe cuando un grupo de edad rechaza el orden existente, se une y trata de redirigir el curso de la política como su misión generacional, pero resulta muy difícil saber cuándo una generación política va a aparecer en la escena política. (GONZALEZ, 2004: 226)

Cuando se dan las circunstancias socio-históricas adecuadas, como el debilitamiento de la autoridad institucional y/o la falta de instrumentos eficaces de socialización, las generaciones históricas pueden implicarse en formas de conflicto intergeneracional a través de la desautorización de forma simbólica o violenta de los valores, normas, prácticas y tradiciones de la vieja generación, y la erección de la juventud en símbolo y vehículo 
del cambio social.

Sin embargo, para Gramsci, citado por GONZALEZ, (2004) las querellas intergeneracionales eran fenómenos superficiales, inherentes a la esfera educativa, a menos que tuvieran un componente clasista, por ejemplo, si los jóvenes de las clases dirigentes se rebelaban y otorgaban su apoyo a la clase progresista. En caso contrario, se produce un estado de rebelión permanente sin una clara salida política, lo que deriva en fenómenos como el misticismo, el sensualismo o la violencia.

\section{DINÁMICAS JUVENILES}

El modelo de generación histórica elaborado por Richard Braungart se presenta como una síntesis alternativa a la visión «constructivista» de la identidad colectiva propia de la teoría generacional clásica, al ahistoricismo de las explicaciones biopsicológicas y al rígido estructuralismo del marxismo, que hace girar el cambio social en torno a conflictos de clase, y del funcionalismo, que considera que la organización de un sistema social está dada de antemano y que la rebeldía juvenil sólo puede pretender un reequilibrio parcial del mismo, según Braungart indica que la protesta juvenil no surge de la nada, sino que es la respuesta a un período de intensa actividad política, fruto de determinados procesos históricos (acontecimientos como crisis sociales, políticas, económicas y culturales, y cambios estructurales como la emigración, la industrialización, la urbanización, la secularización o la burocratización) que interactúan con las fuerzas biológicas y psicológicas para dar lugar a una generación con caracteres propios. (GONZALEZ, 2004:224).

\section{LA PROTESTA JUVENIL}

Desde una perspectiva generacional, la protesta juvenil puede producirse cuando una gran cohorte de edad vive un particular grupo de experiencias históricas, condiciones socio-estructurales y oportunidades para la movilización, generando estructuras propias de movilización.

Los movimientos juveniles vinculados a las generaciones políticas son el resultado de la interacción entre las características del ciclo vital de la juventud (alta energía, conciencia cognitiva, búsqueda de identidad, relaciones entre el yo y la sociedad), las experiencias comunes de la cohorte/generación (crecimiento bajo determinadas condiciones familiares, sociales y globales) y las tendencias sociopolíticas e históricas. (Braungart, 1974; citado por GONZALEZ, 2004)

Los movimientos juveniles expresan insatisfacción respecto del statu quo, y crean de forma revolucionaria nuevas formas de conciencia que son el resultado de fuerzas biológicas, sociales y culturales (Braungart, 1974; citado por GONZALEZ, 2004). Como agentes conscientes del cambio social y político, los movimientos juveniles se comportan en la arena pública de un modo semejante a otros movimientos 
sociales de protesta, y son, por tanto, susceptibles de ser analizados con las herramientas disponibles para el estudio de la acción colectiva, especialmente a través del paradigma de la «estructura de oportunidades políticas».

Doug McAdam enumera tres (3) factores decisivos para la emergencia de un movimiento de protesta. El primero es la expansión de las oportunidades políticas, o elaboración de un contexto favorable para la acción colectiva. Ésta puede entenderse como el fruto de una elección racional basada en el cálculo individual de costos y beneficios, según la tesis clásica de Mancur Olson, los jóvenes están en mejores condiciones objetivas para actuar (es decir, asumen un menor coste de oportunidad política), ya que tienen menos que perder (y, por tanto, aceptan riesgos mayores), no suelen movilizarse con el objetivo exclusivo de obtener incentivos selectivos (sus motivaciones pueden ser más altruistas), y acostumbran a sufrir un nivel de coacción institucional menor (los gobiernos les consideran una «élite protegida», sobre todo si son estudiantes), lo que en su conjunto dificulta el free ridingo comportamiento egoísta que cohíbe la acción. (MacAdam, 1982, citado por GONZALEZ, 2004).

El segundo factor, como advierte McAdam, «entre la oportunidad y la acción median las personas y su forma de entender la situación en la que se encuentran», como un momento intermedio, debe producirse una «liberación cognitiva», o ruptura con la resignación fatalista, que implica la definición de la situación actual como injusta y necesitada de cambio, conforme a reivindicaciones juzgadas como legítimas, y que se acompaña de la convicción de que la acción colectiva puede ser emprendida con perspectivas de éxito.

Esa «liberación cognitiva» está íntimamente ligada a los procesos colectivos de interpretación y comprensión de la realidad, de atribución de significado y de construcción de la identidad colectiva que median entre la oportunidad, la organización y la acción. En este caso, los jóvenes suelen constituir la base de los movimientos sociales de protesta porque crean con gran facilidad identidades y significados comunes con base en los recursos culturales de los que disponen, y ocupan una peculiar posición simbólica en la sociedad, derivada de su participación

en subculturas y estilos de vida que les permiten cuestionar con más eficacia

las normas sociales y reivindicar el derecho a redefinir elecciones vitales (Laraña, 1999, citado por GONZALEZ, 2004).

$\mathrm{Al}$ rechazo en la identificación con el mundo adulto se une la tensión anteel inminente acceso a un mercado laboral cada vez más competitivo, y en el que la integración de las nuevas generaciones resulta en ocasiones muy dificultosa. Los psicólogos sociales destacan la menor adhesión a la autoridad y el afán de libertad y de socialización alternativa de los jóvenes; en una palabra, su idealismo e inconformismo frente al mundo de los adultos al que teóricamente pertenecen. Entienden, como señaló Weber, la política como vocación, como una ética de fines absolutos vinculada a valores como igualdad, honestidad, democracia y socialismo, que en no pocas ocasiones contraponen a actitudes de madurez asociadas a la ética de la responsabilidad.

La tercera condición establecida por McAdam es la capacidad organizativa. No cabe duda de que los vol.10, n. 01, Rio de Janeiro, 2017.pp. 82-94 
jóvenes disponen de recursos organizativos propios, como unas subculturas y unos ámbitos de socialización más cercanos al entorno social básico como son la escuela, el barrio, el club, el grupo de amigos, entre otros.

\section{MODOS GENERALES DE CONFRONTACIÓN POLÍTICA JUVENILES.}

Existen por lo menos cuatro (4) estructuras dominantes de confrontación juvenil a lo largo de la contemporaneidad: la turba estudiantil, la juventud de partido, la organización paramilitar y el movimiento social de nueva generación.

Estas formas organizativas y estos repertorios de acción política violenta de la juventud no han discurrido de forma lineal, sino que conviven, declinan, se reproducen, perviven o se transforman en función del contexto histórico, de los cambios que experimenta el colectivo juvenil en su Weltanschauungy en su propia identidad, y de la estructura de oportunidades políticas. (GONZALEZ, 2004:230).

En cada fase histórica podría constatarse el predominio de una de ellas por su especial eficacia para obtener los objetivos políticos propuestos. De este modo, la protesta escolar fue predominante durante el siglo XIX y la segunda mitad del siglo XX, la violencia partidista y paramilitar tuvieron su época dorada entre el fin de siglo y la Segunda Guerra Mundial, y las reivindicaciones vinculadas a los movimientos sociales de nueva generación han ido adquiriendo protagonismo desde los años 60 hasta la actualidad.

\section{La turba estudiantil}

La movilización escolar es propia de sociedades tradicionales que inician un proceso acelerado de modernización sin alcanzar un alto nivel de implicación política. Un hecho histórico que cataliza una protesta de contenido social o político.

El papel de los estudiantes fue particularmente decisivo en 1830, en 1848, en los movimientos revolucionarios ruso y chino, y en diferentes momentos en Italia, Alemania o España antes de la llegada del fascismo. En esta situación, los escolares (sobre todo los universitarios) se muestran más proclives a tomar parte en movilizaciones de protesta, merced a varias circunstancias concurrentes: el incremento de su importancia numérica y de su influencia social, su autonomía de pensamiento y acción respecto de los grupos sociales y políticos, en el contexto favorable de la autonomía universitaria y de la universidad como centro de pensamiento crítico, foro de discusión y ámbito favorable para la circulación de ideas (BRAUNGART, 1984b:6). Pero en ese tipo de sociedades en proceso de cambio acelerado, la juventud también es particularmente vulnerable a formas radicales de acción política.

La estructura de movilización característica de este tipo de conflictividad es el sindicato profesional (sobre todo el universitario). 


\section{La juventud de partido}

La aparición de los jóvenes como segmento social bien caracterizado a fines de siglo XIX, al hilo de la reducción del porcentaje de población de menor edad en las pirámides de población de los países industrializados, y de la configuración inicial de un mercado juvenil, al que no era ajena la aspiración al «consumo» de «productos» políticos. Entre las instituciones que posibilitaron la aparición de la juventud como grupo social diferenciado figuraban: un modelo nuevo de familia, transformada en ámbito de afectividad y de aprendizaje; la escuela como instrumento normalizado de iniciación social; el ejército como rito de paso, y el mundo laboral como frontera social y económica hacia la edad adulta.

Pero también surgió una legislación social ad hoc, que con el argumento de proteger a la juventud recortó su independencia: tribunales tutelares, servicios de ocupación y bienestar, escuelas, etc. La juventud comenzó a vincularse estrechamente a las luchas políticas.

En ocasiones, la conciencia generacional trató de ser fijada en estructuras orgánicas como las juventudes de partido, aunque una organización juvenil creada y/o liderada por adultos es diferente de otra que es controlada exclusivamente por jóvenes, ya que tiende a quedar supeditada a los criterios políticos de la generación dominante.

\section{La organización paramilitar}

Este tercer tipo de estructura juvenil de confrontación política tuvo su momento de máxima expansión durante el período de entreguerras. La Primera Guerra Mundial abrió las puertas a un sentido juvenil de abordar el arte (vanguardias), la política (radicalismos) o la vida social (inconformismos), marcado por la novedad, la intensidad del compromiso y la amplitud de la movilización.

La dificultosa reintegración de los soldados a la vida civil y la multiplicación de conflictos armados, con la aparición del complejo mundo de las Heimatwehren, guardias cívicas, soviets, ejércitos «rojos» y «blancos», Freikorps, legionarios del Fiume, etc. fueron circunstancias que permitieron a la generación de la guerra y a la juventud que no había sido movilizada instalarse en una vida heroica de violencia antes que en la rutina gris del trabajo en la sociedad civil, a la cual muchos nunca retornaron (LINZ, 1976:34-37).

Los grandes partidos de masas comenzaron a impulsar secciones juveniles pretendidamente autónomas, pero en el contexto de crisis del parlamentarismo liberal, la proliferación de actitudes autoritarias, unida a la proclividad unitarista de la juventud, condujeron a la paulatina emancipación y radicalización de las formaciones juveniles, ya que muchas de ellas se vincularon al sector más extremista, activista y violento de la organización matriz, con la coartada del vanguardismo revolucionario y de la crítica al reformismo.

La Segunda Guerra Mundial marcó el ocaso de esta figura arquetípica del joven como valedor armado de una causa política a través de su encuadramiento en un partido-milicia. 


\section{Los movimientos sociales de nueva generación}

Los nuevos movimientos reivindicativos (asociaciones de consumidores, pacifistas, ecologistas, antinucleares, en pro de los derechos civiles de minorías culturales o raciales ...) tienen un carácter instrumental y una naturaleza marcadamente contracultural; actúan de forma coordinada, en un espacio transnacional y con un elenco de objetivos similares, basados en la reivindicación de derechos. (HABERMAS, 1987: 556.)

El desarrollo de los nuevos movimientos sociales ha generado una nueva cultura del conflicto que es la predominante en la juventud de los países que gozan de amplios e iguales derechos de ciudadanía. (Laraña, 1981, p.386, citado por GONZALEZ, 2004).

Predominan actitudes no violentas, e incluso en su forma más subversiva y militante los nuevos movimientos sociales emplean muy raramente la violencia extrema, y prefieren recurrir a repertorios de acción que utilizan de modo paralelo y flexible formas de acción convencionales (como, por ejemplo, la apelación a los tribunales) y anti-convencionales, basadas en formas no institucionalizadas de participación y de movilización, como las protestas moral o socialmente transgresoras, las manifestaciones festivas y burlescas, las huelgas salvajes, las ocupaciones, los pequeños sabotajes, la desobediencia civil y la insumisión, cuyo componente altamente simbólico y expresivo resulta de gran impacto en la moderna sociedad de la imagen globalizada.

La realidad de una protesta cada vez menos basada en la confrontación física no puede ocultar la realidad de grupos juveniles marginales (como los habitantes de los guetos ciudadanos, los skin-heads, los okupas, los ultras deportivos o los sectores juveniles radicalizados de grupos nacionalistas, separatistas o integristas) que, además de la utilización de modalidades violentas de amplia tradición en la confrontación política, como el terrorismo, la algarada callejera o la agresión individual, emplean una violencia que podríamos definir como «posmoderna»: de baja intensidad, desestructurada, eruptiva, socialmente difusa, escasamente ideologizada y poco discriminada, que a pesar de su limitada capacidad subversiva no ha tenido hasta la fecha una respuesta preventiva o represiva eficaz por parte del Estado.

\section{CONCLUSIONES}

El estudio presentado permitió explorar diferentes elementos que interactúan en procesos de conformación de generaciones políticas. Las especificidades generacionales responden, a las distintas temporalidades que configuran el espacio de una generación. La referencia más importante que forma una generación está ligada a esta conciencia del tiempo vivido, que no es un tiempo neutro sino saturado de experiencias que participan a construir una inteligibilidad política, un tiempo que se vuelve entonces aprendizaje histórico y social que se renueva con el devenir del tiempo.

En lo epistemológico, la perspectiva de Mannheim permite al estudio de lo generacional no sólo abordar vol.10, n. 01, Rio de Janeiro, 2017.pp. 82-94 
las influencias históricas sobre los sujetos coetáneos, sino que también abrir la mirada al estudio de las subjetividades presentes en las conexiones y unidades generacionales.

La falta de renovación (encerramiento) del campo político y los vicios de las formas tradicionales de hacer política devienen escasamente representativas y deprimen el potencial de social de las nuevas generaciones de militantes. La generación de relevo se ubica en una posición marginal dentro del campo político lo que bloquea en parte importante su acceso al poder y favorece su adscripción a las lógicas políticas de los líderes de los nuevos movimientos sociales.

No obstante la generación de relevo tiene potencial de representación ya que se trata de una generación que representa, por una parte la continuidad de los partidos políticos - fueron socializados por sus fundadores y proyectan desde allí sus carreras - pero tienen la distancia crítica, cercanía generacional y vinculación suficiente con la nueva sociedad civil para transitar hacia nuevas prácticas políticas.

\title{
THEORETICAL CONSIDERATIONS POLICIES GENERATIONS
}

\begin{abstract}
This study is based from a sociological and political science perspective theoretically describe different meanings on political generation. In political parties from different countries, it acts the binomial: representative democracy and social participation, showing a weak drivability of organized citizenship, which has resulted in public institutions and political parties lose legitimacy and confidence in citizens, generating internal tensions between young militant leaders of student movements and political parties; these movements lead to a generation that seeks the institutionalization of power, which means an opportunity for renewal of the policy. the hypotheticaldeductive method with the respective literature review for research development is used. Several authors report the concept of generation since the nineteenth century: Comte and Dilthey, Mannheim, and Ortega and Gasset Gramsci. It is concluded that the political generation is characterized by a drastic change in the environment of power relations, affecting or distinctively mark a group with respect to its predecessors, ie, they are contemporary
\end{abstract}

Keywords: Generations Policy Cohorts; Age Groups; Social Participation.

\section{BIBLIOGRAFÍA}

CANO, Jorge. (2015). Política y Generaciones ¿dónde están los jóvenes? Una réplica a Enrique Krauze y a aquellos que juzgan las experiencias políticas de la actual generación de jóvenes mexicanos como "menos válidas" que las de las generaciones anteriores. Fecha: Noviembre 18, 2015. Disponible en: http://horizontal.mx/politicay-generaciones-donde-estan-los-jovenes/\#sthash.T1vwe30b.dpuf

GALAIS, Carolina (2012). "Edad, cohorte o período. Desenredando las causas del desinterés político en España”. Revista Reis 139, julio septiembre 2012, pp. 85-110. Doi: 10.5477/cis/reis.139.85. (España). 
GONZÁLEZ CALLEJA, Eduardo. (2004). "Las jóvenes generaciones contemporáneas. Evolución de los modos conflictivos de participación política”. Mélanges de la Casa de Velázquez, serie 34-1, pp.217-240. (España). Disponible en: https://mcv.revues.org/1195

KOENEKE, H. (2007a). "La protesta estudiantil y el culto a la personalidad de Hugo Chávez Frías". Seminario: Perspectivas Económicas, Políticas y Sociales de Venezuela 2007-2012. Caracas, Junio 28, 2007

Koeneke, H. (2007b). "Información, interés y generaciones políticas". Revista Venezolana Análisis de Coyuntura. Volumen XIII, número 2, pp.367-373.

LANESE, Diego (2016). Militancia universitaria: las nuevas generaciones políticas se abren paso entre el entusiasmo y el prejuicio. Publicado el Miércoles, 25 de mayo de 2016. Disponible en: www.politicadelsur.com

LECCARDI, Carmen; Feixa, Carles (2011). "El concepto de generación en las teorías sobre la juventud”. Revista Última Década. Volumen 19, número 34, Junio 2011, pp. 11-32. (Chile). Disponible en: http://www.scielo.cl/scielo.php?pid=S0718-22362011000100002\&script=sci_arttext

NUNES, R. (2014). “Generación, acontecimiento, perspectiva”. Pensar en el cambio a partir de Brasil”, Nueva Sociedad. Número 251, mayo-junio 2014, pp.42-54.

SUAREZ, Naudy (2007). La Generación del 28 y otras generaciones. Antología de textos. Serie Cuadernos de Ideas Políticas N³. Fundación Rómulo Betancourt.

TIZZIANI, Ania (2008). Generaciones Políticas: una clave de interpretación del largo plazo. Algunas reflexiones a partir del caso del movimiento peronista. Documento de trabajo número 64.

TOLEDO, Giselle Davis (2012). «La generación de recambio político y la nueva sociedad civil: ¿Quién lidera a quién?», Polis [En línea], 32 | 2012, Publicado: 13-12-2012. Consultado: [23-04-2016]. URL: http://polis.revues.org/6497. Doi: 10.4000/polis.6497. (Chile). Disponible en: https://polis.revues.org/6497.

VILLAROEL GLADYS; De Armas, Edoardo. (2005). "Desprecio por la política: aproximación a las representaciones sociales de estudiantes venezolanos" Politeia v.38 n.34-5. Caracas.

YANKELEVICH, Pablo (2011). Pensar la Revolución: Una aproximación a la Generación de 1915. Instituto Nacional de Antropología e Historia. Disponible: http://www.h-mexico.unam.mx/node/6546

Trabalho enviado em 23 de julho de 2016.

Aceito em 14 de setembro de 2016. 